\title{
Experimental study of guiding and filtering of acoustic waves in a two dimensional ultrasonic crystal
}

\author{
Abdelkrim Khelif, Abdelkrim Choujaa, Sarah Benchabane \\ Institut FEMTO-ST, département LPMO, CNRS UMR 6174, \\ 32 avenue de l'Observatoire, F-25044 Besançon, France \\ B. Djafari-Rouhani \\ Laboratoire de Dynamique et Structures des Matériaux Moléculaires, \\ CNRS UMR 8024, Université de Lille I, 59655 Villeneuve d'ascq cedex, France \\ Vincent Laude \\ Institut FEMTO-ST, département LPMO, CNRS UMR 6174, \\ 32 avenue de l'Observatoire, F-25044 Besançon, France
}

\begin{abstract}
We present a combined experimental and theoretical study of the guiding, bending and filtering of acoustic waves in an ultrasonic crystal. The crystal consists of a two-dimensional periodical array of steel rods immersed in water, for wich a complete acoustic band gap extending from 240 to 325 $\mathrm{kHz}$ is found experimentally. Waveguides for acoustic waves are further created by removing a line defect, on which stubs can be added by removing rods from the side-walls of the waveguide. Full transmission is observed for a one-period-wide straight waveguide within the full-band-gap of the perfect phononic crystal, i.e. for a waveguide aperture smaller than one acoustic wavelength. Waveguiding over a wide frequency range is also obtained for a one-period-wide waveguide with two sharp $90^{\circ}$ bends. Finite-difference time-domain computations are found to be in good agreement with the measurements in all experimental configurations.
\end{abstract}

PACS numbers: 46.40.Cd, 63.20.-e, 72.50.+b, 77.65.Dq

\section{INTRODUCTION}

The study of acoustic and elastic wave propagation in periodic band gap materials, known as phononic crystals $[1,2]$, has been receiving a growing interest in recent years. These composite materials are inhomogeneous media constituted of two or three-dimensional arrays of inclusions embedded in a matrix. The band structure of phononic crystals displays absolute gaps provided one imposes a large contrast between the elastic parameters and the mass density of the inclusions and of the matrix.

The existence of absolute band gaps was predicted theoretically and demonstrated experimentally in various phononic crystals constituted of solid components or mixed solid/fluid components [3-9]. In a region of space where a full band gap exists for a given frequency range, waves are unable to propagate anymore. Stated otherwise, only evanescent or inhomogeneous wave solutions are found. This phenomenon can, at least in theory, be considered to occur without attenuation, so that a wave incident on a phononic crystal will be totally reflected to external regions. Conversely, a wave generated in a region of space surrounded by a phononic crystal will be unable to escape from this region. Any defect created within the phononic crystal will permit acoustic energy to propagate again, but only within the defect, leading to interesting properties of spatial confinement, and spectral and temporal filtering in very compact spaces. This makes these systems potential candidates for the design of elastic or acoustic waveguides or filters. Additionally, in the prospect of applications, there is a great deal of interest in developing phononic crystal-based waveguides where one can confine and efficiently guide acoustic waves around sharp corners, which is not feasible with classical waveguides. Also, some degree of control on the spectral characteristics of waveguides is certainly desirable.

The guiding of acoustic waves without losses in straight waveguides using two-dimensional phononic crystals was studied theoretically by several groups [10-12]. However, in contrast to photonic crystals, less attention has been devoted to experimental demonstrations [3, 8, 9], especially for bent waveguides $[3,8,13]$. Waveguides in phononic crystals are produced by removing or changing the cylindrical inclusions along one or several rows of the array. The insertion of the defects, such as cavities, inside or at edge of the waveguide was shown to give rise to the filtering or to the rejection of selective frequencies in the transmission spectrum of the waveguide $[11,14]$.

In this paper, we review and improve our recent experimental results $[9,13]$ with a two dimensional ultrasonic crystal. The geometry of the crystal and the experimental setup are first briefly presented, and some detail is given of the spectral estimation procedures employed. The band gaps of the phononic crystal are then illustrated theoretically and experimentally. Next, the guiding and bending of acoustic waves in highly confined phononic crystal waveguides is investigated. It is shown that a waveguide possesses confined propagation modes within a large frequency range, which led us to further experiments. The calculations throughout the paper are based on the finite difference time domain (FDTD) method that has been proved to be an efficient method 


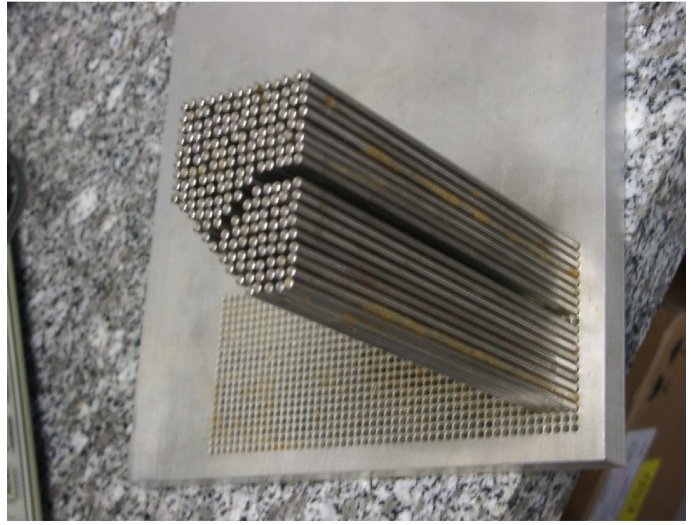

FIG. 1: Phononic crystal constructed by clamping steel cylinders into a rigid periodically perforated steel plate.

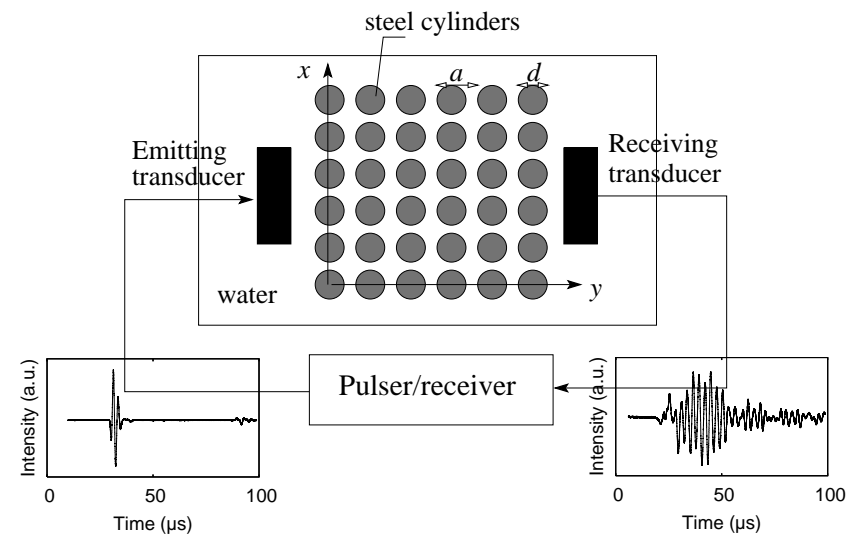

FIG. 2: Experimental set-up used to measure the transmission spectra of phononic crystals using acoustic transducers immersed in water.

for obtaining both the transmission coefficient $[14,16]$ and dispersion curves [15] in phononic crystals.

\section{EXPERIMENTAL SETUP AND GEOMETRICAL PARAMETERS}

We manufactured the 2D lattice of the phononic crystal by clamping $150 \mathrm{~mm}$-long steel cylinders into a rigid periodically perforated steel plate. Figure 1 shows a photograph of one of the tested configurations. The cylinders have a diameter $d=2.5 \mathrm{~mm}$. The period of the square lattice is $a=3 \mathrm{~mm}$. This results in a filling fraction $F=\pi d^{2} /\left(4 a^{2}\right)$ of approximately $55 \%$. The choice of steel and water as the composite materials is based on the strong contrast in their densities and elastic constants.

The measurement setup is based on the well-known ultrasonic immersion transmission technique, depicted in Fig.2. In this technique, a couple of widebandwidth transmitter/receiver acoustic transducers operating around $450 \mathrm{kHz}$ and with a diameter of 25 $\mathrm{mm}$ (Panametrics immersion transducers type Videoscan

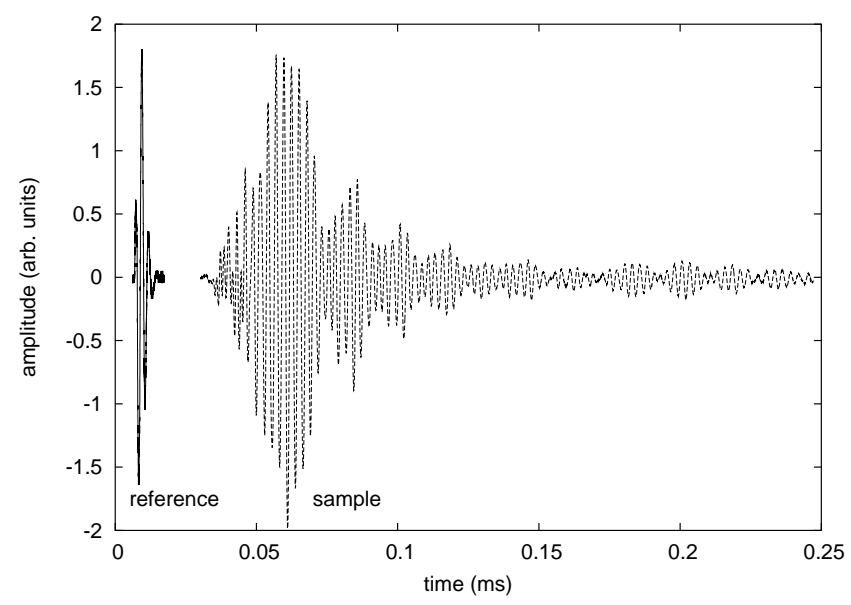

FIG. 3: Examples of temporal signals detected by the receiving transducer without (reference) and with (sample) the phononic crystal.

V301) are used. A pulser/receiver generator (Panametrics model 5800) produces a short duration (about $4 \mu \mathrm{s}$ ) pulse which is applied to the source transducer launching the probing longitudinal waves. The signal detected by the receiving transducer aligned with the source transducer is acquired by the pulser/receiver, post-amplified and then digitized by a digital sampling oscilloscope with a temporal resolution of $2.5 \mathrm{~ns}$. To reduce random errors, 500 measurements are averaged before a Fourier transform is performed to obtain the transmission spectrum. The system is first calibrated with no sample present; a reference signal is digitized and its spectrum is used to normalize the subsequent transmission spectra. Fig. 3 displays the reference and a sample (here a 10-periodlong phononic crystal) temporal signals as detected by the receiving transducer. It can be observed that the dispersion introduced by the phononic crystal is very strong, resulting in a dramatic temporal spreading of the incident acoustic pulse. In comparison with the transmission measurements we published recently $[9,13]$, we have systematically improved the experimental set-up to eliminate parasitic signals, e.g. caused by spurious reflections. As the result, the experimental band-gaps presented here are improved, especially in the spectral regions of low transmission, where parasitic signals are most detrimental.

\section{PHONONIC BAND GAPS}

The band diagram of a phononic crystal gives essential information regarding the propagation of waves within the supposedly infinite crystal. This calculation performed with the FDTD method [15] is shown in Fig. 4 for our square-lattice two-dimensional phononic crystal. Since the Bravais lattice is square and the materials are isotropic, the representation of the bands along the M$\Gamma-\mathrm{X}-\mathrm{M}$ closed path in the first irreducible Brillouin zone 


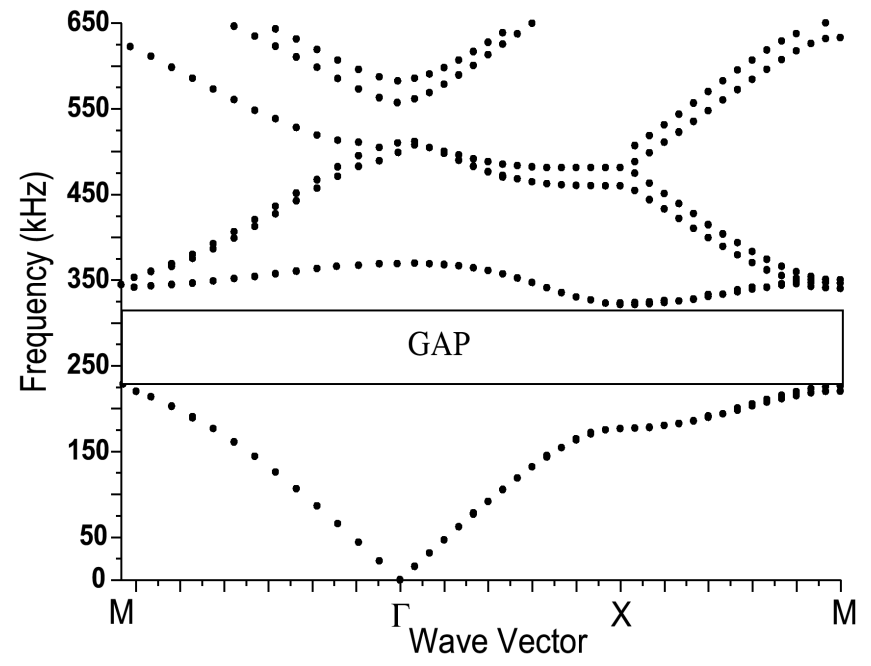

FIG. 4: Band diagram of the phononic crystal.

(shown as an inset in Fig. 4) contains all relevant information. Only the case of longitudinal polarization has to be considered, since only compressional waves exist in the water matrix. Note however that waves of any polarization, i.e. shear and longitudinal, are taken into account within the cylinders. Due to the huge mismatch between the acoustic impedance of steel and water, the steel cylinders act as very efficient scatterers, so that the acoustic energy does not penetrate them notably. As a consequence of scaterring processes, it can be seen that a full band gap extending from 240 to $325 \mathrm{kHz}$ exists.

The effect of the direction of propagation on the band gaps was evaluated experimentally by performing two transmission measurements through the perfect phononic crystal arranged such that the lattice thickness is either parallel to the $\Gamma-\mathrm{X}$ or the $\Gamma-\mathrm{M}$ directions of the square first Brillouin zone, as depicted in Fig. 5. The acoustic transmission spectra of Figs. 5(a) and (b) clearly show a strong attenuation extending from 185 to $325 \mathrm{kHz}$ and from 400 to $487 \mathrm{kHz}$ in the $\Gamma$-X direction, and from 240 to $350 \mathrm{kHz}$ in the $\Gamma-\mathrm{M}$ direction. These observations nicely correlate with the theoretical band structure of Fig. 4. Overlapping these transmission spectra we find a clear proof of the full band gap between 240 and $325 \mathrm{kHz}$ when the two directions are considered jointly. In Fig.5, the computed FDTD transmission spectra are also presented. The location and the width of the band gaps in both directions of propagation compare very well with those observed experimentally.

The origin of band gaps in phononic crystals can be attributed to strong multiple diffraction (scattering) effects. As the crystal is made longer, it is expected that the transmission becomes smaller. Fig. 6 displays the transmission spectra along the $\Gamma-\mathrm{X}$ direction obtained with a 10 -period-long and a 16-period-long phononic crystals. It can be seen that the 6 additional rows yield an additional improvement of $10 \mathrm{~dB}$ close to the high-frequency side of
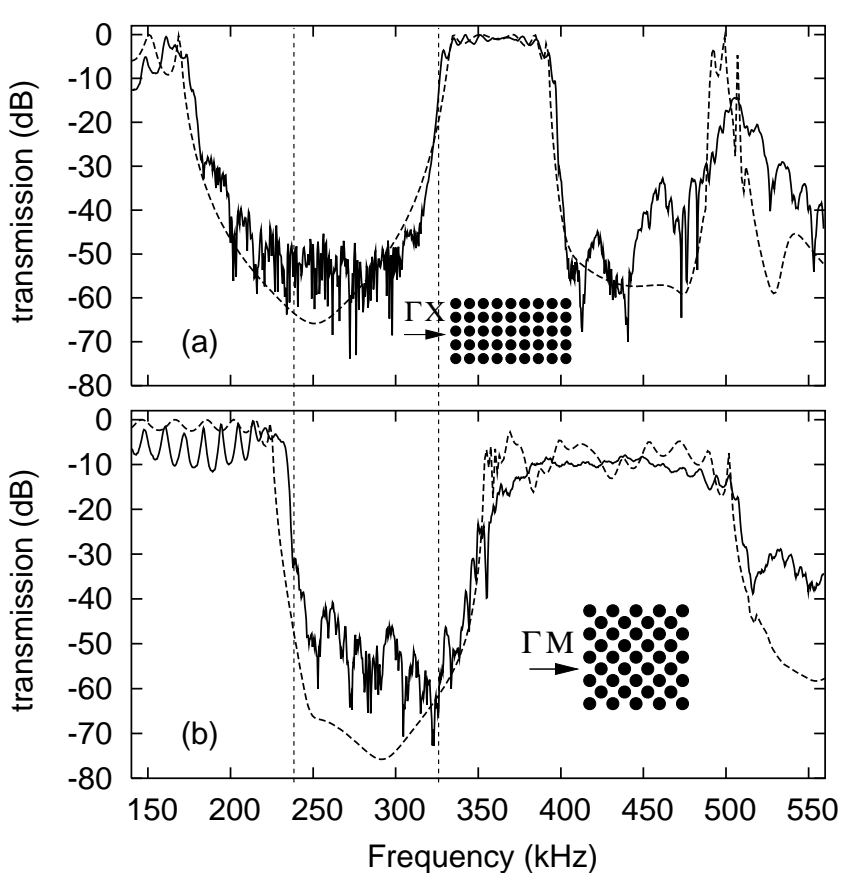

FIG. 5: Experimental (solid lines) and calculated (dashed lines) transmission power spectra along (a) the " $\Gamma X$ " and (b) the " $\Gamma M$ " directions of the irreducible Brillouin zone of the square lattice of steel cylinders in water. A full band gap extending from 250 to $325 \mathrm{kHz}$ is obtained. The insets show the two dimensional cross section of the ultrasonic crystal. Both samples are 10-period-long.

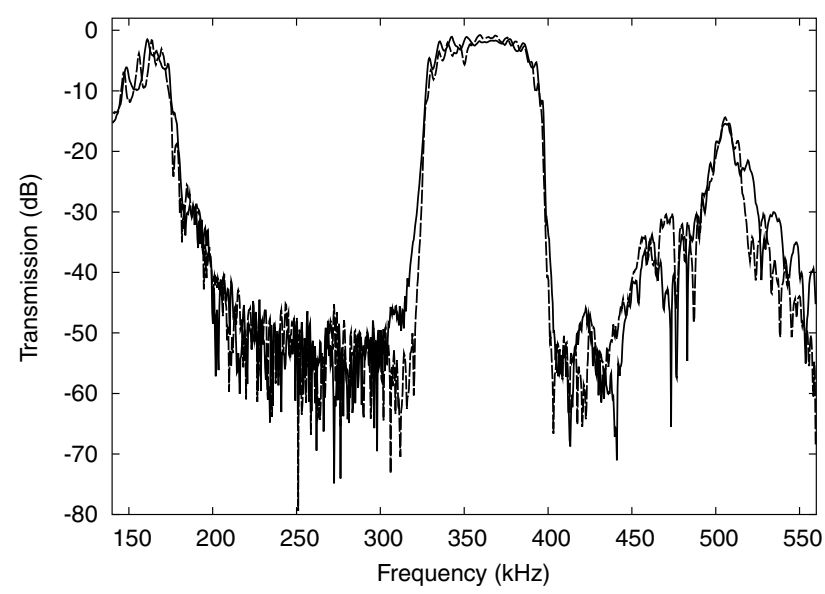

FIG. 6: Experimental transmission power spectra along the $\Gamma$-X direction for a 10-period-long (solid line) and 16-periodlong (dotted line) phononic crystal.

the full band gap (close to $325 \mathrm{kHz}$ ). However, the level of transmission cannot be reduced further by increasing the length of the guide because the noise level in the set-up measurement is reached. More significantly, the transitions between the band gaps and the central passband are made sharper by adding more rows. This is an interesting property for filtering applications where it is 


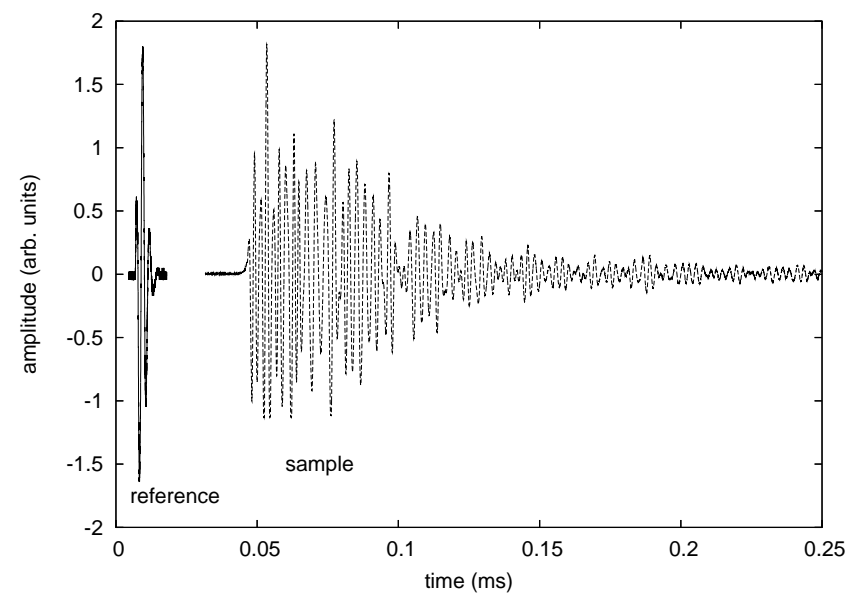

FIG. 7: Examples of temporal signals detected by the receiving transducer without (reference) and with (sample) the waveguide $\mathrm{W} 1$ of the phononic crystal.

important to obtain a square shape spectrum.

\section{GUIDING AND BENDING OF ACOUSTIC WAVES}

In order to achieve a straight waveguide, W1, we remove exactly one row of cylinders along the propagation direction ( $\mathrm{Y}$ axis), as shown in the inset of Fig.8a. The length of waveguide $\mathrm{W} 1$ is 15 periods or $45 \mathrm{~mm}$ and its width, defined by the distance between neighboring cylinders on both sides of the guide, is $3.5 \mathrm{~mm}$. It is worth noting that a classical waveguide of the same width would not be able to propagate waves in the considered frequency range, since its width would be smaller than a wavelength $(\lambda=5.2 \mathrm{~mm}$ at $287 \mathrm{kHz})$. Such a waveguide can only support compressional waves since water admits only longitudinal polarizations. The measured, temporal and spatial transmissions are displayed in Figs.7 and 8a, respectively. Throughout this section, transmission values are normalized with respect to the entrance area of the waveguide. In the numerical calculation, this means that we take the ratio between the outgoing displacement field averaged at the exit of the waveguide and the value of this field in the absence of the sample.

We observe a significant transmission of acoustic waves over a large frequency range within the phononic crystal stop-band. This is strong though indirect indication that the wave is well confined within the waveguide and is guided with weak losses. The guiding band starts at $260 \mathrm{kHz}$ and ends at $315 \mathrm{kHz}$, covering a large part of the stop-band. For comparison, Fig.8a also displays the transmission spectrum for the perfect crystal along the $\Gamma-\mathrm{X}$ axis. The FDTD computation is seen to agree correctly with the measurements.

To obtain a numerical confirmation of the waveguiding properties, the FDTD computation was used to sim-
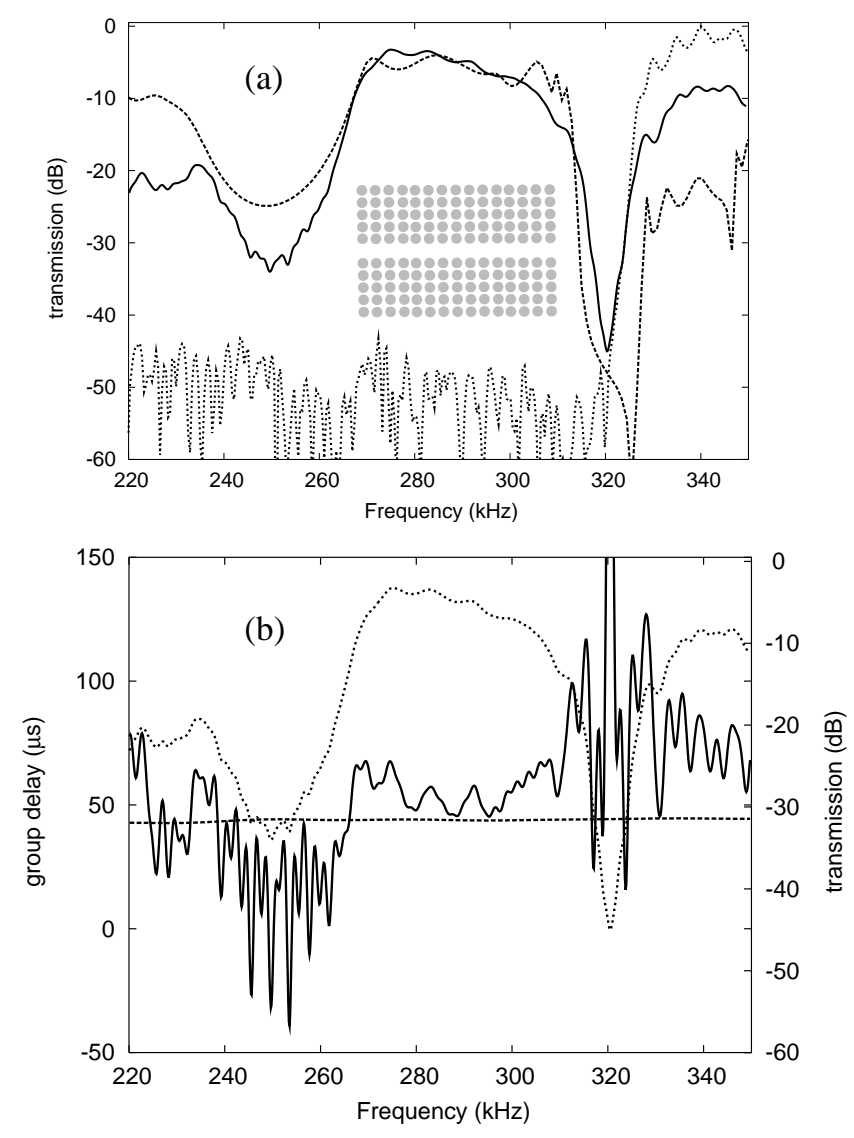

(c)

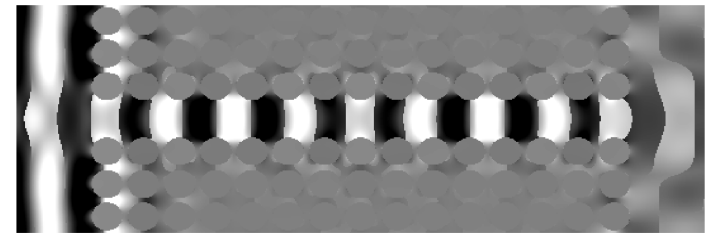

FIG. 8: (a) Experimental (solid line) and calculated (dashed line) transmission power spectra along waveguide W1. The insets show the geometry of the samples. The experimental transmission of the perfect crystal in the $\Gamma$-X direction (dotted line) is shown for comparison. (b) Experimental group delay on transmission though the waveguide (solid line) and on transmission though an equivalent water thickness (dashed line). The transmission power spectrum along waveguide W1 (dotted line) is also shown for comparison. (c) Calculated longitudinal displacements modulus averaged over one period at the frequency $f=287 \mathrm{kHz}$. Grey levels are representative of the amplitude and range from black for negative to white for positive values.

ulate a monochromatic source at frequency $f=287 \mathrm{kHz}$. The computed longitudinal displacements are displayed in Fig. 8c. It is clearly seen that the part of the wave that is incident on the phononic crystal is fully reflected backwards, but that within the waveguide section the wave propagates without attenuation. At the exit of the waveguide, the wave is strongly diffracted since the output aperture is smaller than a wavelength in water.

We also computed from the measurements the group 

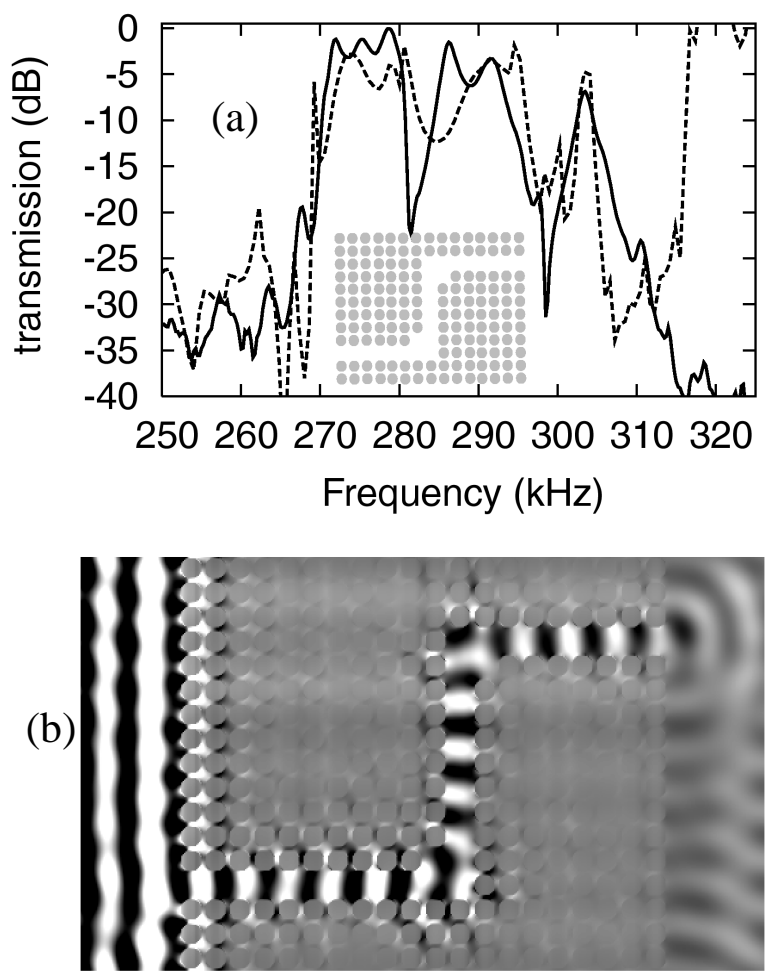

FIG. 9: (a) Same as Fig.7a but for the one-period-wide bended waveguide. (b) Calculated acoustic pressure averaged over one period at the frequency $f=275 \mathrm{kHz}$.

delay on transmission through the waveguide, as depicted in Fig. 8b. The group delay is defined as $t_{g}(\omega)=$ $d \phi(\omega) / d \omega$, where $\phi(\omega)$ is the spectral phase and $\omega$ the angular frequency. As should be expected from the interference of multiply scattered waves [10], the group delay is a rather strongly varying function of frequency. It can be seen anyway that the group delay on transmission through the waveguide, from 260 to $315 \mathrm{kHz}$, is always larger than the group delay on transmission through an equivalent water thickness. This is due to the fact that the group velocity is smaller in the waveguide than in water outside the phononic crystal.

We further tested the bending of acoustic waves through two sharp corners $\left(90^{\circ}\right)$ in the W1 structure shown as an inset in Fig.9a. As shown in Fig.9a, we observe a main waveguiding band extending from 270 to 310 $\mathrm{kHz}$. This spectrum is altered by two transmission drops around $281 \mathrm{kHz}$ and $299 \mathrm{kHz}$. As a result, the whole waveguiding band for the sharply bended structure covers approximately $70 \%$ of the full band gap. Fig.9b is a numerical illustration of the acoustic pressure within the waveguide at $275 \mathrm{kHz}$. The acoustic pressure, $p=\nabla \cdot \mathbf{u}$, includes both the displacements $u_{x}$ and $u_{y}$. The incident wave propagates along the first straight part of the waveguide, successfully couples to the second part with the same shape, although the latter is perpendicular to the former, and again couples to the third part of the waveguide.

\section{CONCLUSION}

In conclusion, we have investigated experimentally and theoretically the band gap properties of a binary twodimensional phononic crystal constituted by a square array of circular parallel steel cylinders in water. We have demonstrated that a fully confined waveguide can be constructed by removing a single row in the phononic crystal. Full transmission of the acoustic wave was obtained through a straight waveguide and a waveguide with a two sharp bends. Potential device applications can be found in the fields of filtering or wavelength demultiplexing for instance.

\section{Acknowledgments}

Fruitful discussions with Marc Solal and Mikael Wilm are gratefully acknowledged. Thanks also go to Rabah Laihem for experimental assistance in the early stage of this work.
[1] M. M. Sigalas and E. N. Economou. Band structure of elastic waves in two dimensional systems. Solid State Commun., 86(3):141-143, 1993.

[2] M. S. Kushwaha, P. Halevi, L. Dobrzynski, and B. Djafari-Rouhani. Acoustic band structure of periodic elastic composites. Phys. Rev. Lett., 71(13):2022-2025, 1993.

[3] F. R. Montero de Espinosa, E. Jimenez, and M. Torres. Ultrasonic band gap in a periodic two-dimensional composite. Phys. Rev. Lett., 80(24):1208-1211, 1998.

[4] V. Sanchez-Perez, D. Caballero, R. Martinez-Sala, C. Rubio, J. Sanchez-Dehesa, F. Meseguer, J. Llinares, and F. Galves. Sound attenuation by a two-dimensional array of rigid cylinders. Phys. Rev. Lett., 80(24):5325-5228, 1998.
[5] D. Caballero, J. Sanchez-Dehesa, C. Rubio, R. MartinezSala, J. V. Sanchez-Perez, F. Meseguer, and J. Llinares. Large two-dimensional sonic band gaps. Phys. Rev. E, 60(6):R6316-R6319, 1999.

[6] I. E. Psarobas, N. Stefanou, and A. Modinos. Phononic crystals with planar defects. Phys. Rev. B, 62(9):55365540, 2000.

[7] J. O. Vasseur, P. A. Deymier, B. Chenni, B. DjafariRouhani, L. Dobrzynski, and D. Prevost. Experimental and theoretical evidence for the existence of absolute acoustic band gaps in two-dimensional solid phononic crystals. Phys. Rev. Lett., 86(14):3012-3015, 2001.

[8] T. Miyashita. Full band gaps of sonic crystals made of acrylic cylinders in air - Numerical and experimental investigations. Jpn. J. App. Phys., 41(5B):3170-3175, 
2002.

[9] A. Khelif, A. Choujaa, B. Djafari-Rouhani, M. Wilm, S. Ballandras, and V. Laude. Trapping and guiding of acoustic waves by defect modes in a full-band-gap ultrasonic crystal. Phys. Rev. B, 68:214301 (4), 2003.

[10] M. Kafesaki, M. M. Sigalas, and N. Garcia. Frequency modulation in the transmittivity of wave guides in elasticwave band-gap material. Phys. Rev. Lett., 85(19):40444047, 2000.

[11] A. Khelif, B. Djafari-Rouhani, J. O. Vasseur, P. A. Deymier, Ph. Lambin, and L. Dobrzynski. Transmittivity through straight and stublike waveguides in a twodimensional phononic crystal. Phys. Rev. B, 65:174308 (5), 2002.

[12] A. Khelif, B. Djafari-Rouhani, J.-O. Vasseur, and P. A. Deymier. Transmission and dispersion relations of perfect and defect-containing waveguide structures in phononic band gap material. Phys. Rev. B, 68:024302 (8), 2003.
[13] A. Khelif, A. Choujaa, S. Benchabane, B. DjafariRouhani, and V. Laude. Guiding and bending of acoustic waves in highly confined phononic crystal waveguides. Appl. Phys. Lett., 84(22):4400-4002, 2004.

[14] M. M. Sigalas and N. Garcia. Theoretical study of three dimensional elastic band gaps with the finite-difference time-domain method. J. Appl. Phys., 87(6):3122-3125, 2000.

[15] Y. Tanaka, Y. Tomoyasu, and S. Tamura Band structure of acoustic waves in phononic lattices: Two-dimensional composites with large acoustic mismatch. Phys. Rev. B,62(11):7387-7392, 2000.

[16] Ph. Lambin, A. Khelif, J. O. Vasseur, L. Dobrzynski, and B. Djafari-Rouhani Stopping of acoustic waves by sonic polymer-fluid composites. Phys. Rev. E,63:066605066605, 2001. 